\title{
Molecular Simulation Study of Dielectric Constants of Pure Fluids and Mixtures
}

\author{
Maximilian Kohns ${ }^{1}$ \\ Laboratory of Engineering Thermodynamics, TU Kaiserslautern, \\ Erwin-Schrödinger Str. 44, D-67663 Kaiserslautern, Germany
}

\begin{abstract}
The static dielectric constant of fluids is studied with molecular models from the literature. The employed molecular models were developed using only vapor-liquid equilibrium data. No information on the dielectric properties was used, so that the simulation results are predictions. A wide range of different fluids, from slightly to strongly polar, is investigated. Most of the studied models underestimate the dielectric constant, which can be explained by the way the models were developed. For the pure fluids dimethyl ether and acetone, the temperature and pressure dependence of the dielectric constant is also studied. A good agreement with experimental data is found. Additionally, binary mixtures are investigated. Thereby, the validity of several mixing rules for the dielectric constant is assessed.
\end{abstract}

Keywords: dielectric constant, relative permittivity, dipolar molecules,

\footnotetext{
${ }^{1}$ Email: maximilian.kohns@mv.uni-kl.de, Telephone: +49-631/205-4028.
} 
property prediction, Kirkwood theory

\section{1. Introduction}

2 The dielectric constant $\varepsilon$, also known as the relative permittivity, charac3 terizes how a certain material weakens the electric field compared to vacuum.

${ }_{4}$ Obviously, it is an important property in electrical engineering. However, the 5 dielectric constant also plays an important role in several process engineer6 ing applications, especially when electrolytes are present. As the dielectric 7 constant directly affects the coulomb potential, it is a key input property

8 for any thermodynamic model for electrolyte solutions, e.g. for $G^{\mathrm{E}}$-models or 9 equations of state.

Due to its utmost importance as a solvent, the dielectric constant of water has been studied in great detail. Lots of experimental data points are available over a wide range of conditions [1]. However, for many other fluids, only few measurements of the dielectric constant have been reported in the literature. In most cases, only state points at or near ambient conditions for temperature and pressure have been studied. While some reports have been made regarding the temperature dependence of the dielectric constant, its pressure dependence has only been studied for few fluids. For many industrially relevant fluids, no experimental data are available at all. 

$36 \quad[8]$.

37 At first, molecular simulations of dielectric constants were carried out in the mixtures. Hence, several different mixing rules for obtaining the dielectric constants of mixtures from the pure fluid dielectric constants have been proposed [2-4], but their range of applicability remains unclear. Thus, a predictive method for obtaining dielectric constants, or at least their temperature, pressure and composition dependence (in case of mixtures), is highly desirable.

Molecular modeling and simulation is a versatile tool for predicting properties of fluids. Molecular models trained to only few experimental data usually allow for making reliable predictions, e.g. at conditions at which experiments are cumbersome or unfeasible. In the light of the Onsager-Kirkwood-Fröhlich theory [5-7], the dielectric constant of a fluid depends on the mutual orientation of the molecular dipole moments. Thus, on the molecular level, the dielectric constant is a structure-related property and is therefore directly accessible in molecular simulations for a given molecular model. It is usually obtained from sampling Kirkwood's formula [6]; however, it has been shown recently that a mapped averaging technique is more accurate at low densities

As a consequence, also few data are available for the dielectric properties of

At first, nholecular sinnulations of diclectric constants were cantied out in the 
framework of developing long-range corrections for electrostatic interactions such as the reaction field method, e.g. by Neumann and co-workers $[9,10]$. Later, molecular simulation results were used to assess perturbation theories for the dielectric [11-15] and to study the dielectric virial series [16, 17]. In most of these studies, Stockmayer or Stockmayer-like fluids, i.e. a LennardJones (LJ) site with a superimposed point dipole, were considered.

Regarding molecular models of real fluids, again water has been studied in detail [18-20]. In addition, also some systematic benchmarks of transferable force fields covering wide ranges of different fluids are available. Caleman et al. [21] presented an evaluation of the OPLS/AA and GAFF force fields and found that most of the models underestimate the dielectric constant. NúñezRojas et al. [22] carried out a similar study on the TraPPE/UA force field and also found that the dielectric constant is typically underestimated, with an average deviation of about 37\%. Zangi [23] investigated the OPLS/AA force field for alcohols, and found an average underestimation of the dielectric constant of about $33 \%$. Including experimental data on the dielectric constant in the model parametrization has been suggested only recently [24, 25].

As all previous systematic benchmarks dealt with transferable force fields, an additional study of essentially independent molecular models for real fluids 
of different polarity is worthwhile. That study is carried out in the present work using the models available in the MolMod database [26]. To this end, molecular models for a wide range of industrially-relevant fluids are taken from the literature and the dielectric constant of the fluid is predicted. The investigated models were developed based on a fit to the experimental vaporliquid equilibrium data (VLE) of the fluid. A comparison to experimental data is carried out to assess how reliable the obtained predictions for the dielectric constant are. In a similar manner, three binary mixtures are considered. This allows to study the physical basis behind mixing rules that have been proposed for the dielectric constant of mixtures.

The paper is structured as follows: In Section 2, the employed molecular models and simulation methods as well as the mixing rules for the dielectric constant are introduced. The results are presented and discussed in Section 3, before summarizing the main conclusions in Section 4.

\section{Methods}

\subsection{Molecular Models}

The MolMod database [26] was searched for molecular models containing either point dipole moments or a partial charge distribution resulting in a 
75

76

77 the dielectric constant was found in the Dortmund Data Bank [27]. Thus,

78

79

80

81

82

83

84

85

86

permanent dipole moment of the entire molecule. 43 models were found. However, for only 25 of these models at least one experimental data point for only those 25 models are considered in the present work. Nevertheless, still a variety of fluids, ranging from slightly to strongly polar, is investigated here. These 25 molecular models were optimized in previous work of our group by a fit to the pure component VLE, hence the dielectric constants studied in the present work are strict predictions obtained from these models. The models are rigid and follow the united-atom approach. The models comprise Lennard-Jones (LJ) sites, point charges, point dipole moments, and point quadrupole moments, so that the total potential energy $U$ in a system of $N$ particles is given by: 


$$
\begin{aligned}
U= & U_{\mathrm{LJ}}+U_{q q}+U_{q \mu}+U_{q Q}+U_{\mu \mu}+U_{\mu Q}+U_{Q Q} \\
= & \sum_{i=1}^{N-1} \sum_{j=i+1}^{N}\left\{\sum_{a=1}^{n_{i}^{\mathrm{LJ}}} \sum_{b=1}^{n_{j}^{\mathrm{LJ}}} 4 \epsilon_{i j a b}\left[\left(\frac{\sigma_{i j a b}}{r_{i j a b}}\right)^{12}-\left(\frac{\sigma_{i j a b}}{r_{i j a b}}\right)^{6}\right]\right. \\
& +\sum_{c=1}^{n_{i}^{e}} \sum_{d=1}^{n_{j}^{e}} \frac{1}{4 \pi \varepsilon_{0}}\left[\frac{q_{i c} q_{j d}}{r_{i j c d}}+\frac{q_{i c} \mu_{j d}+q_{j d} \mu_{i c}}{r_{i j c d}^{2}} f_{1}\left(\omega_{i}, \omega_{j}\right)\right. \\
& +\frac{q_{i c} Q_{j d}+q_{j d} Q_{i c}}{r_{i j c d}^{3}} f_{2}\left(\omega_{i}, \omega_{j}\right)+\frac{\mu_{i c} \mu_{j d}}{r_{i j c d}^{3}} f_{3}\left(\omega_{i}, \omega_{j}\right) \\
& \left.\left.+\frac{\mu_{i c} Q_{j d}+\mu_{j d} Q_{i c}}{r_{i j c d}^{4}} f_{4}\left(\omega_{i}, \omega_{j}\right)+\frac{Q_{i c} Q_{j d}}{r_{i j c d}^{5}} f_{5}\left(\omega_{i}, \omega_{j}\right)\right]\right\}
\end{aligned}
$$

Here, the indices $a, b, c$, and $d$ refer to model interaction sites and $i$ and $j$ refer to molecules, $\varepsilon_{0}$ is the vacuum permittivity, $\epsilon_{i j a b}$ and $\sigma_{i j a b}$ are the LJ energy and size parameters, $r_{i j a b}$ and $r_{i j c d}$ are site-site distances, $q_{i c}$ and $q_{j d}$ are the magnitudes of the point charges, $\mu_{i c}$ and $\mu_{j d}$ are the magnitudes of the point dipoles, $Q_{i c}$ and $Q_{j d}$ are the magnitudes of the point quadrupole moments, and the $f_{k}$ are functions that depend only on the angles $\omega_{i}$ and $\omega_{j}$ that describe the mutual orientation of the molecules $i$ and $j$.

\subsection{Molecular Simulation}

The dielectric constant $\varepsilon$ of pure fluids and mixtures is determined with molecular dynamics (MD) simulations by sampling Kirkwood's formula [6] in the $N p T$ ensemble: 


$$
\varepsilon=1+\frac{\left\langle\boldsymbol{M}^{2}\right\rangle-\langle\boldsymbol{M}\rangle^{2}}{3 \varepsilon_{0} k_{\mathrm{B}} T\langle V\rangle}
$$

${ }_{98}$ Herein, $\boldsymbol{M}$ is the total dipole moment of the simulation box, which is ob-

99 tained by summing up the individual molecular dipole moments $\boldsymbol{\mu}_{i}$ :

$$
\boldsymbol{M}=\sum_{i} \boldsymbol{\mu}_{i}
$$

100

The other symbols in Eq. (2) have their usual meaning: $k_{\mathrm{B}}$ is Boltzmann's constant, $T$ is the temperature, and $\langle V\rangle$ is the $N p T$ ensemble average of the volume. As only isotropic, non-ferroelectric fluids are studied in the present work, the term $\langle\boldsymbol{M}\rangle^{2}$ in Eq. (2) vanishes.

All MD simulations of the present work were carried out using an extended version of the program $m s 2$ [28]. Further simulation details are documented in the Appendix.

\subsection{Mixing Rules for the Dielectric Constant}

Looyenga [3] presented a simple empirical mixing rule that directly yields the dielectric constant of the mixture:

$$
\varepsilon=\left(\sum_{k} \phi_{k} \varepsilon_{k}^{1 / 3}\right)^{3}
$$


110

112 fraction of $j$ in the mixture, defined as

$$
\phi_{j}=\frac{x_{j} v_{j}}{\sum_{k} x_{k} v_{k}}
$$

${ }_{113}$ where $v_{k}$ is the molar volume of pure $k$. Oster [2] and Wang and Anderko ${ }_{114}[4]$ developed mixing rules based on the polarisation of the mixture. In both 115 cases, first the polarisation $P_{k}$ of all pure components is evaluated as

$$
P_{k}=\frac{\left(\varepsilon_{k}-1\right)\left(2 \varepsilon_{k}+1\right)}{9 \varepsilon_{k}} .
$$

116 Then, the polarisation of the mixture $P$ is computed. In the case of Oster's 117 rule it is given by

$$
P=\rho \sum_{k} x_{k} v_{k} P_{k}
$$

${ }_{118}$ where $\rho$ is the density of the mixture. In the case of Wang and Anderko's 119 rule, the polarisation of the mixture is computed as

$$
P=\sum_{k} \phi_{k} P_{k}
$$


120 are obtained from molecular simulations. For pure fluids, for each of the studied molecular models, the relative deviation

$$
\Delta \varepsilon=\frac{\varepsilon^{\operatorname{Sim}}-\varepsilon^{\operatorname{Exp}}}{\varepsilon^{\operatorname{Exp}}}
$$

131

The only difference between Eqs. (6) and (7) is that the rule by Wang and Anderko neglects the excess volume and therefore relies solely on pure component data. Oster's rule considers the excess volume, meaning that e.g. the density $\rho$ of the mixture must be known. Finally, for both rules the dielectric constant of the mixture is evaluated by solving the equation for the polarisation of the mixture

$$
P=\frac{(\varepsilon-1)(2 \varepsilon+1)}{9 \varepsilon}
$$

for $\varepsilon$.

\subsection{Objectives}

In the present work, the dielectric constants of pure fluids and mixtures between the molecular simulation prediction of the dielectric constant $\varepsilon^{\operatorname{Sim}}$ and the experimental data from the literature $\varepsilon^{\text {Exp }}$ is assessed. For each fluid, one statepoint close to ambient temperature and pressure is consid- 
ered, as experimental data are available only at such conditions for many of the studied fluids. All statepoints correspond to liquids. For several fluids, the pressure was not reported with the experimental data. In these cases, a pressure somewhat larger than the vapor pressure of the fluid at the investigated temperature was chosen for the simulations. For two fluids, namely dimethyl ether and acetone, experimental data are available for a wide range of temperatures and pressures, so that these two fluids are studied in more detail.

Also binary mixtures are considered in the present work. All three binary systems comprising the components water, methanol and acetone are investigated. This choice is again mainly motivated by the availability of experimental data. Moreover, these systems are interesting as highly non-ideal dielectric mixing effects are observed. As before, the employed molecular models are taken from the literature, namely SPC/E for water [29], the methanol model by Schnabel et al. [30], and the acetone model by Windmann et al. [31]. The interactions between unlike LJ sites are described by the Lorentz-Berthelot combining rules 


$$
\begin{aligned}
\sigma_{i j a b} & =\frac{\sigma_{i i a a}+\sigma_{j j b b}}{2}, \\
\epsilon_{i j a b} & =\sqrt{\epsilon_{i i a a} \epsilon_{j j b b}} .
\end{aligned}
$$

151

In addition to the comparison of molecular simulation predictions to experimental data, also the mixing rules introduced in the previous section are assessed in the present work.

\section{Results and Discussion}

\subsection{Pure Fluids}

The results of the systematic study of 25 molecular models are compiled in Table 1 and are shown as a histogram of deviations in Figure 1. [Table 1 about here.]

[Figure 1 about here.]

The histogram shows that the molecular models typically underestimate the dielectric constant. The majority of the models underestimate the dielectric constant by 25 to $75 \%$. The absolute average deviation is about $50 \%$. One likely reason is the model parametrization: The models were trained to VLE 
data only. Typically, in that adjustment the magnitude of the point dipole or the charge distribution, respectively, are used as adjustable parameters. Training the model to VLE data means that a trade-off between liquid and gas phase properties must be made. Consequently, the magnitude of the resulting dipole moment of the molecule is somewhat lower than might be expected for a liquid state. As the dipole moment enters quadratically in the Kirkwood equation (2), a slightly too low dipole moment automatically results in a large underestimation of the dielectric constant. This hypothesis is supported by the results obtained for acetonitrile. For this fluid, two different molecular models are available. Still, both were trained to the same VLE data, and their deviation of the dielectric constant is similar.

The present simulation results also allow for a discussion if an assignment of partial charges or the use of a point dipole is more appropriate for describing the dielectric constant. From the 25 models, 17 employ a point dipole, and 8 employ a distribution of partial charges (cf. last column of Table 1). When comparing their deviations to experimental data, however, no systematic trend is observed, i.e. the models using partial charges do not outperform the ones using point dipole moments (or vice versa). Hence, the general choice whether to use a point dipole or a partial charge distribution does not affect 
the ability of the molecular model to predict the dielectric constant.

Another likely reason for the underestimation of the dielectric constant is the neglect of polarizability, which is common to most molecular models available to date. This hypothesis is supported by the fact that several transferable force fields also underestimate the dielectric constant [21-23].

The systematic study shows that molecular models trained to VLE data can usually not be used for accurate quantitative predictions of dielectric constants. However, as has already been demonstrated for water [32], dedicated molecular models can of course be adjusted with respect to the dielectric constant. If adjusted to data at only one statepoint, these models are potentially useful for predicting e.g. the temperature and pressure dependence of the dielectric constant. To test this hypothesis, fortunately, for two of the fluids studied here experimental data are available over a wide range of temperatures and pressures. These fluids are dimethyl ether and acetone. At the statepoint investigated thus far the deviations for these two fluids were $26 \%$ and only $1 \%$, respectively. This should be kept in mind when looking at the predictions for other conditions.

Figure 2 shows the dielectric constant of dimethyl ether for several isotherms as a function of the pressure. The numerical simulation results are docu- 
mented in Table 2.

[Figure 2 about here.]

[Table 2 about here.]

The molecular model for dimethyl ether overestimates the dielectric constant at all temperatures, but the deviations decrease with increasing temperature. Thus, the influence of temperature on the dielectric constant is underestimated by the model. However, the pressure dependence is predicted in excellent agreement with the experimental data as the deviations are independent of the pressure.

Figure 3 shows one isobar and one isotherm of the dielectric constant of acetone. The numerical simulation results are documented in Table 3.

[Figure 3 about here.]

[Table 3 about here.]

The molecular model of acetone, which already showed excellent agreement with the experimental data at the single state point considered above, performs excellently for both the temperature and the pressure dependence of the dielectric constant. The model yields reliable predictions even at very 
high pressures, where experimental investigations are difficult. Thus, in this case molecular modeling and simulation is an excellent tool for obtaining thermodynamic properties at extreme conditions.

Altogether, the results of the present study suggest that a molecular model that captures dielectric constant at one statepoint accurately can also be used to describe the pressure and temperature dependence of the dielectric constant accurately.

\subsection{Mixtures}

Figure 4 shows the dielectric constants of the three binary mixtures of the components water, methanol and acetone as a function of the composition. In addition to the experimental and molecular simulation data, also values obtained from the mixing rules introduced in Section 2.4 are included. To stress the deviation from 'ideal' dielectric mixing behavior, also the excess dielectric constant $\varepsilon^{\mathrm{E}}$, defined as

$$
\varepsilon^{\mathrm{E}}=\varepsilon-\sum_{k} x_{k} \varepsilon_{k}
$$

is shown. The numerical simulation results are compiled in Table 4. 
[Table 4 about here.]

The molecular simulation predictions for the dielectric constants in the studied binary mixtures are not perfect. However, this is mainly due to the inaccurate description of the dielectric constants of pure water and methanol: The excess dielectric constants are in good agreement with the experimental data. This is a remarkable finding as the dielectric mixing in the investigated systems is highly non-ideal: The excess dielectric constant has a large contribution; it is on the order of $50 \%$ of the total value in the water-acetone system and about $10 \%$ for the other systems. Thus, molecular simulations can serve as a predictive tool to study dielectric constants of mixtures quantitatively if models are available that capture the pure fluids' dielectric constants quantitatively.

As the molecular models intrinsically capture the unlike electrostatics in a physical way, the obtained simulation results can be used as an excellent benchmark for assessing mixing rules for the dielectric constant. Thus, the simulation results for the pure fluids were used to evaluate the mixing rules by Looyenga, Oster, and Wang and Anderko for all three binary systems. For the evaluation of Oster's rule, additionally the densities of the mixtures are needed, which are, however, readily available from the $N p T$ simulations. 
For the system water-methanol, the mixing rules by Oster and by Wang and Anderko, which have some physical reasoning, perform much better than the rather empirical correlation by Looyenga. Surprisingly, this is not the case for the water-acetone system, for which Looyenga's rule is in best agreement with the molecular simulation data. For the system methanol-acetone, clearly all three mixing rules fail to describe the dielectric mixing excess that is found in the molecular simulation data. The simulations are able to predict the non-ideality, even though the dielectric constants of the two pure fluids are predicted to be almost equal. For the systems investigated here, this comparison shows that molecular simulation is a much more reliable tool for studying dielectric constants of mixtures than the use of mixing rules.

\section{Conclusions}

Dielectric constants of pure fluids and binary mixtures are computed with molecular dynamics simulations. A comprehensive study of molecular models from the literature that were optimized to VLE properties is carried out, considering one statepoint for each fluid. The majority of the investigated molecular models underpredicts the dielectric constant, with an average deviation of about $50 \%$. However, this disagreement with experimental data can 
be attributed to the model parametrization. When developing a model to describe the entire fluid region, compromises must be made between properties of liquid, gaseous, and supercritical states. The resulting dipole moments are typically somewhat lower than expected for a liquid, which automatically leads to an underestimation of the dielectric constant in the light of Kirkwood's theory. Nevertheless, for the two fluids acetone and dimethyl ether, for which more experimental data are available for comparison, a study of other statepoints reveals that the temperature and pressure dependence is predicted in good agreement with the experimental trends. Thus, molecular models can serve as a reliable tool to extrapolate the dielectric behavior to state conditions that are difficult or even impossible to investigate experimentally.

An application to binary mixtures of water, methanol and acetone shows that molecular models, although they might disagree with experimental data for the dielectric constants of the pure components, predict the excess dielectric constant, i.e. the effect of mixing, well. They are thus reliable predictive tools for obtaining dielectric properties e.g. of mixed solvents in electrolyte systems. Furthermore, although giving the correct trends for some systems, all the investigated empirical mixing rules for the dielectric constant have a 
less sound physical basis than the molecular models.

\section{Acknowledgments}

The present work was conducted under the auspices of the BoltzmannZuse Society of Computational Molecular Engineering (BZS) and the simulations were carried out on the Regional University Computing Center Kaiserslautern (RHRK) under the grant TUK-TLMV, the High Performance Computing Center Stuttgart (HLRS) under the grant MMHBF2 as well as the Leibniz Supercomputing Centre (LRZ) under the grant (AMSEL)² (pn56mo). I thank Jadran Vrabec, Kai Langenbach and Hans Hasse for fruitful discussions.

\section{Appendix A. Simulation Details}

In the present work, MD simulations were carried out with the molecular simulation program $m s 2$ [28]. Equilibration and production took 500,000 and 10,000,000 time steps of length $1.2 \mathrm{fs}$, respectively. The simulations were run with a total of 1000 particles in the $N p T$ ensemble, employing the velocity scaling thermostat, Andersen's barostat, and a LJ cutoff radius of $17.5 \AA$ for the simulations of pure components and $15.0 \AA$ for the simulations 
of mixtures. Statistical simulation uncertainties were estimated with the block average method by Flyvbjerg and Petersen [33]. Long-range corrections to the LJ interactions were considered by the angle-averaging approach by Lustig [34]. Electrostatic long-range interactions were calculated using the reaction field method with conducting boundary conditions, i.e. assuming an infinite dielectric constant of the continuum outside the reaction field sphere. This choice has no influence on the simulation result for the dielectric constant of the fluid under investigation [10]. Additionally, for two models, namely the SPC/E water model and the methanol model by Schnabel et al., the simulations of the dielectric constants at the statepoint investigated in the main text were repeated using Ewald summation instead. The results using these two independent methods were in mutual agreement.

\section{References}

[1] D. Fernandez, A. Goodwin, E. Lemmon, J. Levelt Sengers, R. Williams, A formulation for the static permittivity of water and steam at temperatures from $238 \mathrm{~K}$ to $873 \mathrm{~K}$ at pressures up to $1200 \mathrm{MPa}$, including derivatives and Debye-Hückel coefficients, J. Phys. Chem. Ref. Data 26 (1997) 1125-1166. 
${ }_{327}^{22}[2]$ G. Oster, The Dielectric Properties of Liquid Mixtures, $328 \quad$ J. Am. Chem. Soc. 68 (1946) 2036-2041.

329 [3] H. Looyenga, Dielectric Constants of Homogeneous Mixtures, Mol. Phys. $330 \quad 9(1965) 501-511$.

331 [4] P. Wang, A. Anderko, Computation of dielectric constants of solvent $332 \quad$ mixtures and electrolyte solutions, Fluid Phase Equilib. 186 (2001) 103$333 \quad 122$

${ }_{334}^{34}[5]$ L. Onsager, Electric Moments of Molecules in Liquids,

[9] M. Neumann, O. Steinhauser, The influence of boundary conditions used 
in machine simulations on the structure of polar systems, Mol. Phys. 39 (1980) 437-454.

[10] M. Neumann, O. Steinhauser, G. Stuart Pawley, Consistent calculation of the static and frequency-dependent dielectric constant in computer simulations, Mol. Phys. 52 (1984) 97-113.

[11] D. Boda, J. Liszi, I. Szalai, Preliminary communication: Dielectric constant of a stockmayer fluid along the vapour-liquid coexistence curve, Mol. Phys. 85 (1995) 429-434.

[12] D. Boda, J. Winkelmann, J. Liszi, I. Szalai, Vapour-liquid equilibrium of Stockmayer fluids in applied field: Application of the NpTE plus test particle method and perturbation theory, Mol. Phys. 87 (1996) 601-624.

[13] V. Kalikmanov, Algebraic perturbation theory for polar fluids: A model for the dielectric constant, Phys. Rev. E 59 (1999) 4085-4090.

[14] M. Valisko, D. Boda, Relative permittivity of polar liquids, comparison of theory, experiment, and simulation, J. Phys. Chem. B 109 (2005) 6355-6365.

[15] K. Langenbach, M. Kohns, Relative Permittivity of Dipolar Model Flu- 
ids from Molecular Simulation and from the Co-Oriented Fluid Functional Equation for Electrostatic Interactions, J. Chem. Eng. Data, in press.

[16] K. Morozov, The dielectric virial expansion and the models of dipolar hard-sphere fluid, J. Chem. Phys. 126 (2007) 3849.

[17] S. Yang, A. Schultz, D. Kofke, Evaluation of second and third dielectric virial coefficients for non-polarisable molecular models, Mol. Phys. 115 (2017) 991-1003.

[18] L. MacDowell, C. Vega, Dielectric Constant of Ice Ih and Ice V: A computer simulation study, J. Phys. Chem. B 114 (2010) 6089-6098.

[19] J. Aragones, L. MacDowell, C. Vega, Dielectric constant of ices and water: A lesson about water interactions, J. Phys. Chem. A 115 (2011) $5745-5758$.

[20] O. Gereben, L. Pusztai, On the accurate calculation of the dielectric constant from molecular dynamics simulations: The case of SPC/E and SWM4-DP water, Chem. Phys. Lett. 507 (2011) 80-83.

[21] C. Caleman, P. Van Maaren, M. Hong, J. Hub, L. Costa, D. van der 
Spoel, Force field benchmark of organic liquids: Density, enthalpy of vaporization, heat capacities, surface tension, isothermal compressibility, volumetric expansion coefficient, and dielectric constant, J. Chem. Theory. Comput. 8 (2012) 61-74.

[22] E. Núñez-Rojas, J. Aguilar-Pineda, A. Pérez De La Luz, E. de Jesús González, J. Alejandre, Force Field Benchmark of the TraPPE-UA for Polar Liquids: Density, Heat of Vaporization, Dielectric Constant, Surface Tension, Volumetric Expansion Coefficient, and Isothermal Compressibility, J. Phys. Chem. B 122 (5) (2018) 1669-1678, cited By 4.

[23] R. Zangi, Refinement of the OPLS/AA Force-Field for Liquid Alcohols, ACS Omega 3 (2018) 18089-18099.

[24] F. Salas, G. Méndez-Maldonado, E. Núñez-Rojas, G. Aguilar-Pineda, H. Domínguez, J. Alejandre, Systematic procedure to parametrize force fields for molecular fluids, J. Chem. Theory Comput. 11 (2015) 683-693.

[25] J. Méndez-Bermúdez, H. Dominguez, L. Pusztai, S. Guba, B. Horváth, I. Szalai, Composition and temperature dependence of the dielectric 
constant of 1-propanol/water mixtures: Experiment and molecular dynamics simulations, J. Mol. Liq. 219 (2016) 354-358.

[26] S. Stephan, M. Horsch, J. Vrabec, H. Hasse, MolMod - an open access database of force fields for molecular simulations of fluids, Mol. Sim. 45 (2019) 806-814.

[27] DDBST GmbH, Dortmund Data Bank, Version 2016, Oldenburg, 2016.

[28] G. Rutkai, A. Köster, G. Guevara-Carrion, T. Janzen, M. Schappals, C. Glass, M. Bernreuther, A. Wafai, S. Stephan, M. Kohns, S. Reiser, S. Deublein, M. Horsch, H. Hasse, J. Vrabec, ms2: A molecular simulation tool for thermodynamic properties, release 3.0, Comput. Phys. Commun. 221 (2017) 343-351.

[29] H. Berendsen, J. Grigera, T. Straatsma, The Missing Term in Effective Pair Potentials, J. Phys. Chem. 91 (1987) 6269-6271.

[30] T. Schnabel, A. Srivastava, J. Vrabec, H. Hasse, Hydrogen bonding of methanol in supercritical $\mathrm{CO}_{2}$ : Comparison between ${ }^{1} \mathrm{H}$ NMR spectroscopic data and molecular simulation results, J. Phys. Chem. B 111 (2007) 9871-9878. 
411

412

413

414

415

416

417

418

419

420

421

422

423

424

425

426

427

[31] T. Windmann, M. Linnemann, J. Vrabec, Fluid Phase Behavior of Nitrogen plus Acetone and Oxygen plus Acetone by Molecular Simulation, Experiment and the Peng-Robinson Equation of State, J. Chem. Eng. Data 59 (2014) 28-38.

[32] R. Fuentes-Azcatl, J. Alejandre, Non-polarizable force field of water based on the dielectric constant: TIP4P/ع, J. Phys. Chem. B 118 (2014) $1263-1272$.

[33] H. Flyvbjerg, H. Petersen, Error-estimates on Averages of Correlated Data, J. Chem. Phys. 91 (1989) 461-466.

[34] R. Lustig, Angle-average for the powers of the distance between two separated vectors, Mol. Phys. 65 (1988) 175-179.

[35] W. Eltringham, O. J. Catchpole, Relative permittivity measurements of gaseous, liquid, and supercritical dimethyl ether, J. Chem. Eng. Data 52 (2007) 363-367.

[36] B. Eckl, J. Vrabec, H. Hasse, Set of molecular models based on quantum mechanical ab initio calculations and thermodynamic data, J. Phys. Chem. B 112 (2008) 12710-12721. 
[37] J. Gmehling, T. Teutenberg, P. Wagner, AiF-Project Rep. Nr. 14514 N (2008).

[38] Y. Uosaki, S. Kitaura, T. Moriyoshi, Static relative permittivities of water plus acetone and water plus dimethyl sulfoxide under pressures up to $300 \mathrm{MPa}$ at $298.15 \mathrm{~K}$, J. Chem. Eng. Data 42 (1997) 580-584.

[39] D. Jannakoudakis, G. Papanastasiou, P. Mavridis, Epistemonike Epeteris 15 (1975) 101-117.

[40] A. Campbell, E. Kartzmar, Thermodynamic and other Properties of Methanol + Acetone, Carbon Disulfide + Acetone, Carbon Disulfide + Methanol, and Carbon Disulfide + Methanol + Acetone, J. Chem. Thermodyn. 5 (1973) 163-172.

[41] K. Fredenhagen, Z. Phys. Chem. (Leipzig) 128 (1927) 1-24.

[42] T. Merker, J. Vrabec, H. Hasse, Molecular simulation study on the solubility of carbon dioxide in mixtures of cyclohexane+cyclohexanone, Fluid Phase Equilib. 315 (2012) 77-83.

[43] J. Nath, S. Mishra, Fluid Phase Equilib. 145 (1998) 89-97.

[44] T. Schnabel, M. Cortada, J. Vrabec, S. Lago, H. Hasse, Molecular model 
for formic acid adjusted to vapor-liquid equilibria, Chem. Phys. Lett. 435 (2007) 268-272.

[45] A. Lutskii, S. Mikhailenko, J. Struct. Chem. USSR 4 (1963) 12-14.

[46] G. Moumouzias, D. Panopoulos, G. Ritzoulis, J. Chem. Eng. Data 36 (1991) 20-23.

[47] S. Deublein, Molecular Modeling and Simulation of Electrolyte Solutions, Ph.D. thesis, University of Kaiserslautern (2012).

[48] Y.-L. Huang, M. Heilig, H. Hasse, J. Vrabec, Vapor-Liquid Equilibria of Hydrogen Chloride, Phosgene, Benzene, Chlorobenzene, OrthoDichlorobenzene, and Toluene by Molecular Simulation, AIChE J. 57 (2011) 1043-1060.

[49] N. Sastry, R. Thakor, M. Patel, J. Mol. Liq. 144 (2009) 13-22.

[50] H. Schlundt, A. Germann, J. Phys. Chem. 29 (1925) 353-355.

[51] T. Merker, G. Guevara-Carrión, J. Vrabec, , H. Hasse, Molecular Modeling of Hydrogen Bonding Fluids: New Cyclohexanol Model and Transport Properties of Short Monohydric Alcohols, in: Nagel, W.E and 
Kröner, D.B. and Resch, M.M.: High Performance Computing in Science and Engineering '08, 2009.

[52] V. Durov, N. Lifanova, A. Artykov, Zh. Fiz. Khim. 59 (1985) 2043-2044.

[53] D. Gill, J. Solution Chem. 8 (1979) 691-699.

[54] N. Gee, J.-P. Shinsaka, K.and Dodelet, J. Chem. Thermodyn. 18 (1986) $221-234$.

[55] F. Linde, Ann. Physik 292 (1895) 546-570.

[56] M. Zafarani-Moattar, H. Shekaari, J. Chem. Thermodyn. 38 (2006) 624633.

[57] C. Snyder, J. Douglas, J. Phys. Chem. B 104 (2000) 11058-11065.

[58] T. Schnabel, J. Vrabec, H. Hasse, Molecular simulation study of hydrogen bonding mixtures and new molecular models for mono- and dimethylamine, Fluid Phase Equilib. 263 (2008) 144-159.

[59] P. Le Fevre, R.J.W. Russell, Trans. Faraday. Soc. 43 (1947) 374-393.

[60] E. Elts, T. Windmann, D. Staak, J. Vrabec, Fluid phase behavior from molecular simulation: Hydrazine, Monomethylhydrazine, Dimethylhy- 
477 drazine and binary mixtures containing these compounds, Fluid Phase 478 Equilib. $322(2012)$ 79-91.

479

480

481

482

483

484

485

486

487

488

489

490

491

492

493

[61] H. Ulich, W. Nespital, Z. Phys. Chem. Abt. B 16 (1932) 221-233.

[62] T. Schnabel, J. Vrabec, H. Hasse, Henry's law constants of methane, nitrogen, oxygen and carbon dioxide in ethanol from 273 to $498 \mathrm{~K}$ : Prediction from molecular simulation, Fluid Phase Equilib. 233 (2005) 134-143.

[63] A. Chmielewska, M. Zurada, K. Klimaczewski, A. Bald, J. Chem. Eng. Data 54 (2009) 801-806.

[64] N. Sastry, R. Thakor, M. Patel, Int. J. Thermophys. 54 (2008) 801-806.

[65] B. Eckl, Y.-L. Huang, J. Vrabec, H. Hasse, Vapor pressure of R227ea+ethanol at $343.13 \mathrm{~K}$ by molecular simulation, Fluid Phase Equilib. 260 (2007) 177-182.

[66] A. Ribeiro, C. Nieto de Castro, R. Pai-Panandiker, U. Mardolcar, J. Chem. Eng. Data 52 (2007) 2041-2049.

[67] N. Gee, G. Freeman, J. Chem. Thermodyn. 25 (1993) 549-554.

[68] R. Holland, C. Smyth, J. Phys. Chem. 59 (1955) 1088-1092. 
${ }_{494}$ [69] B. Eckl, J. Vrabec, H. Hasse, An optimised molecular model for ammo495 nia, Mol. Phys. 106 (2008) 1039-1046.

496 [70] G. Billaud, A. Demortier, J. Phys. Chem. 79 (1975) 3053-3055.

497 [71] W. Harder, E. Franck, Z. Phys. Chem. (München) 161 (1989) 123-130.

${ }_{498}[72]$ B. Eckl, J. Vrabec, H. Hasse, On the application of force fields for pre499 dicting a wide variety of properties: Ethylene oxide as an example, Fluid $500 \quad$ Phase Equilib. 274 (2008) 16-26.

501 [73] P. Walden, Z. Phys. Chem. (Leipzig) 70 (1910) 569-619. 


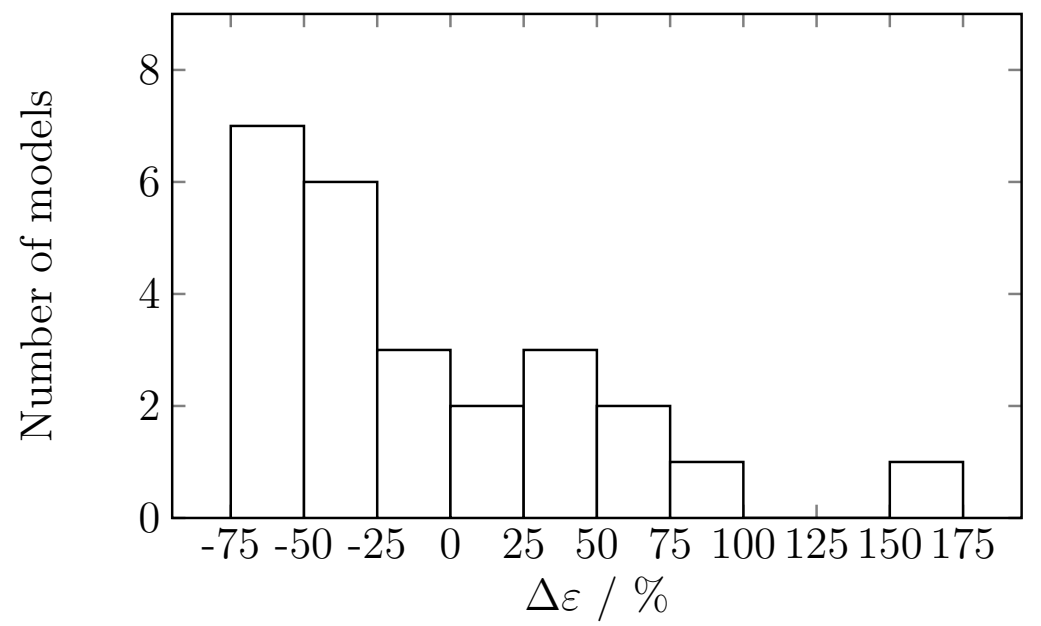

Figure 1: Histogram of deviations $\Delta \varepsilon$ between experiment and molecular simulations for the 25 investigated molecular models. 

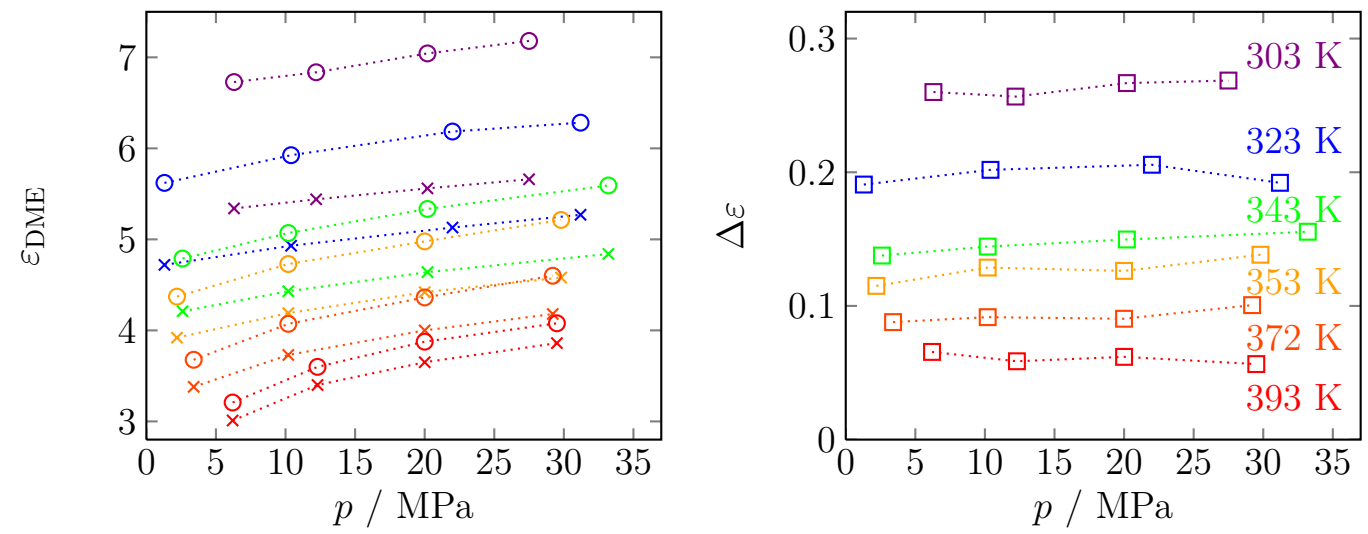

Figure 2: Isotherms of the dielectric constant of dimethyl ether over the pressure. Left: $(\times)$ Experimental data [35], ( $)$ molecular simulation predictions using the model by Eckl et al. [36]. Right: Deviation in the dielectric constant. Dotted lines are guides to the eye. 

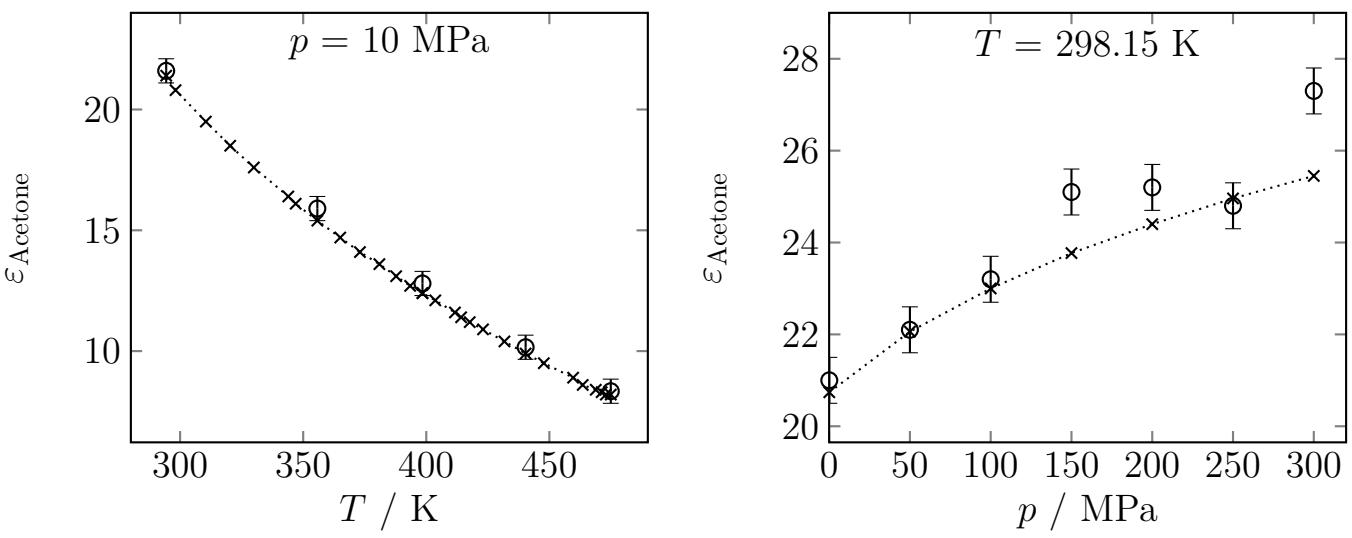

Figure 3: $10 \mathrm{MPa}$ isobar (left) and $298.15 \mathrm{~K}$ isotherm (right) of the dielectric constant of acetone. $(\times)$ Experimental data [37, 38], ( $)$ molecular simulation predictions using the model by Windmann et al. [31]. Dotted lines are guides to the eye. 

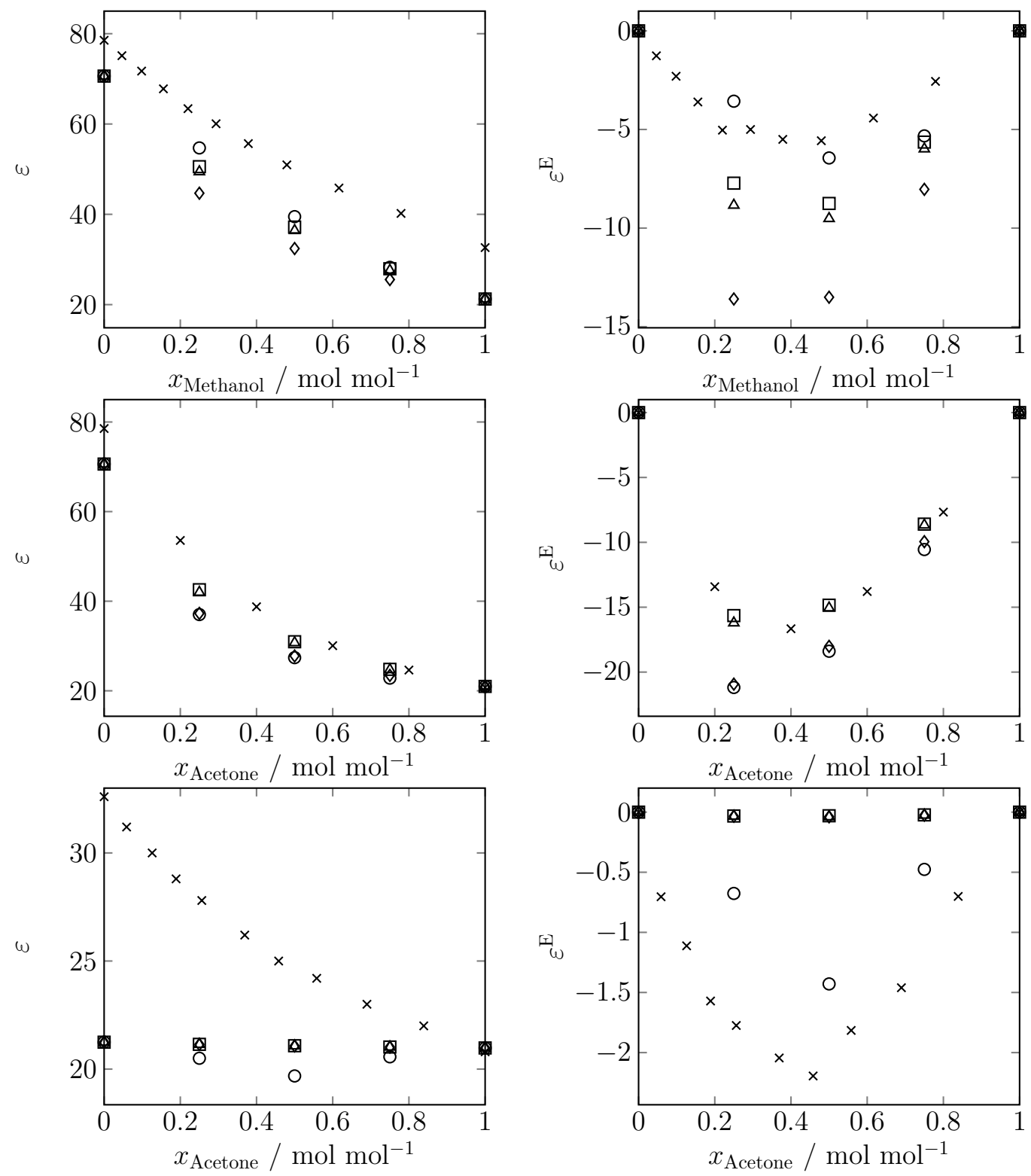

Figure 4: Dielectric constants (left panels) and excess dielectric constants (right panels) of binary mixtures at $298.15 \mathrm{~K}$ and 1 bar. Top row: water-methanol, middle row: water-acetone, bottom row: methanol-acetone. $(\times)$ Experimental data [38-40], (०) molecular simuation predictions. Predictions from mixing rules evaluated with the dielectric constants of the pure fluids from simulation: $(\square)$ Oster [2], $(\triangle)$ Wang-Anderko [4], $(\diamond)$ Looyenga [3]. 
Table 1: Dielectric constant of 25 pure fluids at the indicated statepoints from experiment and molecular simulation. The entries are sorted by ascending deviation $\Delta \varepsilon$ between simulation and experiment. The experimental data were retrieved from the Dortmund Data Bank [27]. For the simulation results, statistical uncertainties for the last significant digit are given in parentheses. The last column indicates if the model uses a distribution of partial charges (entry $q$ ) or a point dipole moment (entry $\mu$ ).

\begin{tabular}{lrcrrrrrr}
\hline \hline Fluid & Model Ref. & $T / \mathrm{K}$ & $p / \mathrm{MPa}$ & $\varepsilon^{\text {Exp }}$ & Exp. Ref. & $\varepsilon^{\text {Sim }}$ & $\Delta \varepsilon$ & Model Type \\
\hline \hline Hydrogen Cyanide & {$[36]$} & 295.85 & 1.0 & 111.000 & {$[41]$} & $31.5(3)$ & -0.72 & $\mu$ \\
Cyclohexanone & {$[42]$} & 303.15 & 0.1 & 17.950 & {$[43]$} & $5.0(1)$ & -0.72 & $\mu$ \\
Formic Acid & {$[44]$} & 298.15 & 0.1 & 51.100 & {$[45]$} & $14.7(4)$ & -0.71 & $q$ \\
Acetonitrile & {$[36]$} & 298.15 & 0.1 & 35.990 & {$[46]$} & $12.5(2)$ & -0.65 & $\mu$ \\
Acetonitrile & {$[47]$} & 298.15 & 0.1 & 35.990 & {$[46]$} & $13.9(2)$ & -0.61 & $\mu$ \\
Toluene & {$[48]$} & 298.15 & 0.1 & 2.408 & {$[49]$} & $1.109(2)$ & -0.54 & $\mu$ \\
Phosgene & {$[48]$} & 295.15 & 1.0 & 4.368 & {$[50]$} & $2.04(1)$ & -0.53 & $\mu$ \\
Cyclohexanol & {$[51]$} & 298.00 & 0.1 & 16.400 & {$[52]$} & $8.4(4)$ & -0.49 & $q$ \\
Nitromethane & {$[36]$} & 298.15 & 0.1 & 36.700 & {$[53]$} & $20.8(4)$ & -0.43 & $\mu$ \\
Isobutane & {$[36]$} & 296.00 & 1.0 & 1.727 & {$[54]$} & $1.01(1)$ & -0.42 & $\mu$ \\
Sulfur Dioxide & {$[36]$} & 296.15 & 1.0 & 14.800 & {$[55]$} & $8.91(5)$ & -0.40 & $\mu$ \\
Methanol & {$[30]$} & 298.15 & 0.1 & 32.730 & {$[56]$} & $21.2(7)$ & -0.35 & $q$ \\
o-Dichlorobenzene & {$[48]$} & 293.24 & 0.1 & 10.177 & {$[57]$} & $7.40(2)$ & -0.27 & $\mu$ \\
Methylamine & {$[58]$} & 298.15 & 1.0 & 9.400 & {$[59]$} & $8.09(6)$ & -0.14 & $q$ \\
Hydrazine & {$[60]$} & 298.15 & 0.1 & 51.700 & {$[61]$} & $45(2)$ & -0.13 & $q$ \\
Ethanol & {$[62]$} & 298.15 & 0.1 & 24.350 & {$[63]$} & $21.6(8)$ & -0.11 & $q$ \\
Acetone & {$[31]$} & 298.15 & 0.1 & 20.740 & {$[38]$} & $21.0(3)$ & 0.01 & $\mu$ \\
Chlorobenzene & {$[48]$} & 298.15 & 0.1 & 5.698 & {$[64]$} & $7.0(3)$ & 0.23 & $\mu$ \\
Dimethyl Ether & {$[36]$} & 303.70 & 6.3 & 5.340 & {$[35]$} & $6.71(4)$ & 0.26 & $\mu$ \\
R227eaa & {$[65]$} & 293.14 & 1.0 & 4.097 & {$[66]$} & $5.50(8)$ & 0.34 & $\mu$ \\
Dimethyl Sulfide & {$[36]$} & 296.30 & 0.1 & 6.511 & {$[67]$} & $9.7(1)$ & 0.49 & $\mu$ \\
Thiophene & {$[36]$} & 293.15 & 0.1 & 2.769 & {$[68]$} & $4.27(3)$ & 0.54 & $\mu$ \\
Ammonia & {$[69]$} & 298.15 & 2.0 & 16.030 & {$[70]$} & $24.7(1)$ & 0.54 & $q$ \\
Hydrogen Chloride & {$[48]$} & 288.15 & 10.0 & 4.960 & {$[71]$} & $9.84(3)$ & 0.98 & $q$ \\
Ethylene Oxide & {$[72]$} & 272.15 & 0.1 & 13.900 & {$[73]$} & $35.5(4)$ & 1.55 & $\mu$ \\
\hline \hline & & & & & & & & \\
\end{tabular}

a) 1,1,1,2,3,3,3-Heptafluoropropane 
Table 2: Molecular simulation results for the dielectric constant of dimethyl ether using the model by Eckl et al. [36]. Statistical uncertainties for the last significant digit are given in parentheses.

\begin{tabular}{cccccc}
\hline \hline$p / \mathrm{MPa}$ & $\varepsilon$ & $p / \mathrm{MPa}$ & $\varepsilon$ & $p / \mathrm{MPa}$ & $\varepsilon$ \\
\hline \hline \multicolumn{2}{c}{$T=303 \mathrm{~K}$} & \multicolumn{2}{c}{$T=323 \mathrm{~K}$} & \multicolumn{2}{c}{$T=343 \mathrm{~K}$} \\
6.3 & $6.73(4)$ & 1.3 & $5.62(3)$ & 2.6 & $4.79(2)$ \\
12.2 & $6.84(4)$ & 10.4 & $5.72(3)$ & 10.2 & $5.07(2)$ \\
20.2 & $7.04(4)$ & 22.0 & $6.18(3)$ & 20.2 & $5.33(3)$ \\
27.5 & $7.18(4)$ & 31.2 & $6.28(3)$ & 33.2 & $5.59(3)$ \\
$T=353 \mathrm{~K}$ & $T=372 \mathrm{~K}$ & $T=393 \mathrm{~K}$ \\
2.2 & $4.37(2)$ & 3.4 & $3.68(1)$ & 6.2 & $3.21(1)$ \\
10.2 & $4.73(2)$ & 10.2 & $4.07(2)$ & 12.3 & $3.60(1)$ \\
20.0 & $4.98(2)$ & 20.0 & $4.36(2)$ & 20.0 & $3.88(1)$ \\
29.8 & $5.21(3)$ & 29.2 & $4.60(2)$ & 29.5 & $4.08(2)$ \\
\hline \hline
\end{tabular}


Table 3: Molecular simulation results for the dielectric constant of acetone using the model by Windmann et al. [31]. Statistical uncertainties for the last significant digit are given in parentheses.

\begin{tabular}{lccc}
\hline \hline \multicolumn{2}{c}{$p=10 \mathrm{MPa}$} & \multicolumn{2}{c}{$T=298.15 \mathrm{~K}$} \\
\hline \hline$T / \mathrm{K}$ & $\varepsilon$ & $p / \mathrm{MPa}$ & $\varepsilon$ \\
294 & $21.6(3)$ & 0.1 & $21.0(3)$ \\
355 & $15.9(2)$ & 50 & $22.1(3)$ \\
398 & $12.8(1)$ & 100 & $23.2(4)$ \\
440 & $10.16(8)$ & 150 & $25.1(4)$ \\
474 & $8.34(6)$ & 200 & $25.2(5)$ \\
& & 250 & $24.8(5)$ \\
& & 300 & $27.3(6)$ \\
\hline \hline
\end{tabular}


Table 4: Molecular simulation results for the dielectric constant of binary mixtures of water $(\mathrm{W})$, methanol $(\mathrm{MeOH})$, and Acetone $(\mathrm{AC})$ at $298.15 \mathrm{~K}$ and 1 bar. The composition is given as the mole fraction of component 2. Statistical uncertainties for the last significant digit are given in parentheses.

\begin{tabular}{lcrr}
\hline \hline$x_{2} / \mathrm{mol} \mathrm{mol}^{-1}$ & $\mathrm{~W}(1)-\mathrm{MeOH}(2)$ & $\mathrm{W}(1)-\mathrm{AC}(2)$ & $\mathrm{MeOH}(1)-\mathrm{AC}(2)$ \\
\hline \hline 0 & $70(2)$ & $70(2)$ & $21.2(7)$ \\
0.25 & $55(2)$ & $37.0(8)$ & $20.5(4)$ \\
0.5 & $39(1)$ & $27.4(5)$ & $19.7(3)$ \\
0.75 & $28.2(9)$ & $22.8(3)$ & $20.6(3)$ \\
1 & $21.2(7)$ & $21.0(3)$ & $21.0(3)$ \\
\hline \hline
\end{tabular}

